\title{
Individualized Homeopathic Medicines in Chronic Rhinosinusitis: Randomized, Double-Blind, Placebo-Controlled Trial
}

\author{
Pankhuri Misra ${ }^{1}$ Chintamani Nayak ${ }^{1}$ Abhijit Chattopadhyay ${ }^{1}$ Tarun Kumar Palit ${ }^{2}$ Bharti Gupta ${ }^{3}$ \\ Satarupa Sadhukhan ${ }^{4}$ Koushik Bhar ${ }^{1}$ Shruti Rai ${ }^{4}$ Maneet Parewa ${ }^{5}$ Sk. Swaif Ali ${ }^{6}$ Anamika Basu ${ }^{6}$ \\ Arunava Nath ${ }^{4}$ Munmun Koley ${ }^{7}$ Subhranil Saha ${ }^{8}$
}

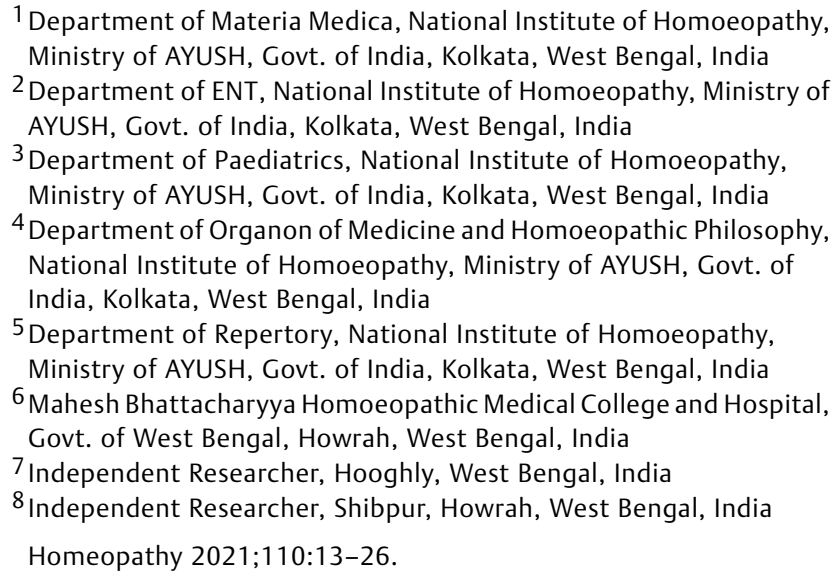

Address for correspondence Pankhuri Misra, Department of Materia Medica, National Institute of Homoeopathy, Ministry of AYUSH, Govt. of India, Block GE, Sector III, Salt Lake, Kolkata 700 106, West Bengal, India (e-mail: drpankhuri7hc@gmail.com).

\begin{abstract}
Keywords

- efficacy

- homeopathy

- chronic rhinosinusitis

- sino-nasal outcome test

- randomized controlled trial

Background Chronic rhinosinusitis (CRS) is a common disorder, with up to an estimated 134 million Indian sufferers, and having significant impact on quality of life (QOL) and health costs. Despite the evidence favoring homeopathy in CRS being inadequate, it is highly popular. This trial attempts to study the efficacy of individualized homeopathy $(\mathrm{IH})$ medicines in comparison with placebo in patients with CRS.

Methods Adouble-blind, randomized (1:1), placebo-controlled, preliminary trial $(n=62)$ was conducted at the National Institute of Homoeopathy, West Bengal, India. Primary outcome measure was the sino-nasal outcome test-20 (SNOT-20) questionnaire; secondary outcomes were the EQ-5D-5L questionnaire and EQ-5D-5L visual analog scale scores, and five numeric rating scales $(0-10)$ assessing intensity of sneezing, rhinorrhea, post-nasal drip, facial pain/pressure, and disturbance in sense of smell, all measured at baseline and after the 2 nd and 4th months of intervention. Group differences and effect sizes (Cohen's $d$ ) were calculated on the intention-to-treat sample.

Results Groups were comparable at baseline. Attrition rate was 6.5\% (IH: 1, Placebo: 3). Although improvements in both primary and secondary outcome measures were higher in the $\mathrm{IH}$ group than placebo, with small to medium effect sizes, the group differences were statistically non-significant (all $p>0.05$, unpaired $t$-tests). Calcarea carbonica, Lycopodium clavatum, Sulphur, Natrum muriaticum and Pulsatilla nigricans were the most frequently prescribed medicines. No harmful or unintended effects, homeopathic aggravations or any serious adverse events were reported from either group.
\end{abstract}

received

March 29, 2020

accepted after revision

May 26, 2020

published online

August 31, 2020 (c) 2020. The Faculty of Homeopathy.

All rights reserved.

Georg Thieme Verlag KG,

Rüdigerstraße 14,

70469 Stuttgart, Germany
DOI https://doi.org/

10.1055/s-0040-1715842. ISSN 1475-4916. 
Conclusion There was a small but non-significant direction of effect favoring homeopathy, which ultimately renders the trial as inconclusive. Rigorous trials and independent replications are recommended to arrive at a confirmatory conclusion. [Trial registration: CTRI/2018/03/012557; UTN: U1111-1210-7201].

\section{Introduction}

Chronic rhinosinusitis (CRS) is one of the most common chronic and debilitating conditions affecting 10 to $15 \%$ of the world population. ${ }^{1,2}$ An estimated 134 million Indians suffer from CRS. ${ }^{3}$ CRS is defined as a symptomatic inflammation of the paranasal sinuses and nasal cavity persisting for more than 12 weeks. In 1996, the American Academy of OtolaryngologyHead \& Neck Surgery (AAO-HNS) multidisciplinary Rhinosinusitis Task Force defined adult rhinosinusitis diagnostic criteria, which were updated by AAO-HNS in $2015 .{ }^{4}$ According to the recent update, 12 weeks or longer with two or more of the signs and symptoms, such as muco-purulent drainage (anterior, posterior, or both), nasal obstruction (congestion), facial pain-pressure-fullness, or disturbed sense of smell, are indicative of CRS. ${ }^{1}$ Inflammation is documented by one or more of the findings such as purulent mucus or edema in the middle meatus or anterior ethmoid region, polyps in the nasal cavity or the middle meatus, and/or radiographic imaging showing inflammation of the paranasal sinuses. ${ }^{3}$ It has been shown to impair the quality of life (QOL) and increase the risk of depression, anxiety, and mood and sleep disorders. ${ }^{5-7}$ Thus, CRS is frequently linked with significant morbidity and health care expenditure. ${ }^{8}$ The economic impact has been estimated as quite cumbersome annually, when direct and indirect costs are taken into account. ${ }^{9,10}$

Current management techniques are mainly targetted at reducing inflammation with the goal of restoring normal sinus physiology, and consist of antibiotics, nasal decongestants, topical nasal steroids and/or oral steroids, saline irrigation, and surgery, ${ }^{11}$ but the results are highly variable. ${ }^{12}$ According to the National Ambulatory Medical Care Survey, sinusitis is the fifth most common diagnosis treated with antibiotics. ${ }^{1,13}$ An earlier Cochrane review concluded that antibiotics provide a minor improvement in simple (uncomplicated) sinus infections; however, the small benefit gained may be overridden by the negative effects of antibiotics, both on the patient and on the population in general. ${ }^{14}$ In a recent Cochrane Review, it was shown that only 5 out of 100 patients treated with antibiotics recovered faster, while these patients experienced significantly more adverse events than those receiving placebo. ${ }^{15}$ Despite appropriate medical therapy, a subset of patients with CRS continues to be symptomatic and eventually undergoes endoscopic sinus surgery. ${ }^{16}$

Due to this disease, there is significant patient morbidity in terms of negatively affected QOL and substantial impairment of daily functioning, resulting in decreased overall productivity. Many continue to suffer and seek other treatments because the relapse rate can be as high as $47.5 \%$. In a cohort study, patients with CRS were twice as likely as the general population to enquire about complementary and alternative medicine (CAM). ${ }^{17} \mathrm{Up}$ to $79 \%$ of individuals in developed countries ${ }^{18,19}$ and $40 \%$ of patients with CRS in North America ${ }^{20-22}$ have reported using at least one CAM therapy. Estimates show that $50 \%$ or more of adults in the United States and all over the world turn to alternative therapies to treat chronic conditions including CRS. ${ }^{23}$ Some alternative therapies show promise as potential treatments for CRS, compared with placebo or as adjuncts to usual care, but based on the limited quality data there is scarce evidence to support the use of homeopathy in CRS. ${ }^{24}$

Homeopathy research on sinusitis comprises both acute and chronic conditions. No systematic reviews or metaanalysis could be identified on the subject. Two randomized controlled trials (RCTs) on two different homeopathic complex remedies reported significant reduction in symptoms of acute rhinosinusitis (ARS) compared with placebo. ${ }^{25,26} \mathrm{Sev}-$ en more non-randomized trials of different homeopathic complexes in ARS were identified. ${ }^{27-33}$ In an open, nonrandomized, observational study of Sinuforce spray in 81 patients with either ARS or CRS, $72 \%$ of patients rated the treatment as "very good" or "good" 34 . In CRS, two doubleblind RCTs, in 173 and 152 patients respectively, were identified: one compared a homeopathic complex Euphorbium comp. S against placebo ${ }^{35}$; in the other, three non-individualized, standardized remedies-Luffa operculata D4, Kalium bichromicum D4 and Cinnabaris D3-were compared against placebo. ${ }^{36}$ Whilst the former trial demonstrated a significant superiority of the verum against placebo and was well tolerated, the latter one revealed no differences amongst groups. In an open, randomized, parallel arm trial of a complex remedy $D r$. Reckeweg $R 1$ in 38 patients suffering from CRS, the treatment group receiving amoxicillin/clavulanic acid plus Dr Reckeweg $R 1$ drops showed a statistically significant improvement of all clinical parameters related to a control group that was untreated. ${ }^{37}$

Effects of the classical or individualized form of homeopathy has remained unexplored through RCTs. Even after a careful search, we could identify only four open-label, single arm, observational studies of individualized homeopathy $(\mathrm{IH})$ in $\mathrm{CRS}^{38-41}$; all suggested promising treatment effects, reduced symptom intensity, and improved QOL, thereby indicating a need to conduct RCTs of IH in CRS. Recently, however, the Central Council for Research in Homoeopathy (CCRH) has initiated an efficacy trial of IH treatment, using 50 millesimal potencies versus placebo, in patients suffering from CRS; ${ }^{42}$ the trial is ongoing and the results are yet to be published.

The current work sought to assess the efficacy of IH in centesimal scale through a double-blind, randomized, 
placebo-controlled design. We hypothesized that IH plus general management, over 2 and 4 months of intervention, might act differently from identical looking placebo plus general management as reflected in sino-nasal outcome test20 (SNOT-20) scores $^{43}$ in patients suffering from CRS. We also aimed at shortlisting the most frequently prescribed $\mathrm{IH}$ medicines in CRS.

\section{Methods}

Trial design: This randomized, double-blind, placebo-controlled, two parallel arms, preliminary clinical trial was conducted at the Materia Medica and Ear-Nose-Throat (ENT) out-patient departments of the National Institute of Homoeopathy (NIH), under the Ministry of AYUSH, Govt. of India (Kolkata, West Bengal). The study protocol was approved by the Institutional Ethics Committee (IEC; Ref. no. 523/NIH/PG/Ethical Comm. 2009/Vol. 5/ 2588 (A/S); dated March 6, 2018) and was registered prospectively in the Clinical Trials Registry-India (CTRI/2018/03/012557) prior to enrollment of the patients. It also had a secondary identifier (Universal trial number) of U1111-1210-7201. The trial protocol and full dissertation were submitted as the postgraduate thesis of the corresponding author to the West Bengal University of Health Sciences (Kolkata, West Bengal, India).

Participants: Inclusion criteria were patients suffering from CRS for 12 weeks or more (2020 ICD-10-CM diagnosis code J32.9), who had not taken any treatment for the last 2 weeks, fulfilled the diagnostic criteria of AAO-HNS, were aged between 18 and 60 years and of either sex, could read Bengali, gave written consent to participate in the study, and were willing to take only homeopathic medicines. Exclusion criteria were: suffering from uncontrolled systemic illness or life-threatening infections; suffering with complications of CRS; insisting on or in need of surgical interventions; already undergoing homeopathic treatment for any chronic disease; substance abuse and/or dependence; pregnant or lactating women; patients with psychiatric disease; and those with a self-reported immune-compromised state.

Intervention: Patients were divided into two parallel arms:

1. Experimental arm: Intervention was planned as administering indicated homeopathic medicines in centesimal potencies as per the need of the patient and as decided appropriate by the homeopaths. Each dose of centesimal scale consisted of 6 to 8 cane sugar globules no. 10 medicated with the indicated medicine (preserved in $90 \% \mathrm{v} / \mathrm{v}$ ethanol), which was taken orally on a clean tongue and with empty stomach. The dosage and repetition depended upon the individual requirement of the case. Those in the intervention arm were allowed the option of taking saline steam inhalations and paracetamol as rescue medication, if and when required. Other medicines such as antibiotics, glucocorticosteroids and anti-allergics were not permitted. The duration of therapy for each patient was 4 months. All medicines were procured from a Good Manufacturing Practice-certified firm (Hahnemann Publishing Company Private Ltd., Kolkata, West
Bengal, India). Final selection of the single individualized medicine and dosage was in accordance with standard homeopathic guidelines and by agreement among three homeopaths. The prescriptions on follow-up visits were generated as per relevant homeopathic principles and were recorded in follow-up sheets. Homeopathic prescriptions were permitted to change during the study as and when required in adherence with homeopathic principles. Two of the prescribers possessed a master's degree in homeopathy, with more than 15 years of experience of teaching and practicing classical homeopathy; the other prescriber was one of six postgraduate trainees at NIH. All the homeopaths involved were affiliated with state councils.

2. Comparator arm: These patients received placebo, indistinguishable from verum. Each dose of placebo was taken orally on a clean tongue and with empty stomach. This group was also allowed to take saline steam inhalations and paracetamol, if required, but other medicines such as antibiotics, glucocorticosteroids and anti-allergics were not permitted. The duration of the study for each patient was 4 months. As in the verum group, patients in the control arm were assessed by the three homeopaths. The nature of the medicine (whether verum or placebo) was blinded for the patient, prescribing doctors, and outcome assessor.

General management: All the patients were encouraged to avoid alcohol and tobacco, or a dusty environment, to prevent any aggravation. They were advised to maintain a regular and nutritious diet. They were recommended saline steam inhalation to get temporary relief of the troublesome symptoms. In cases of any acute conditions or adverse events, provision was made to treat the patients with acute homeopathic medicines and to revert to the assigned coded treatment once the acute condition had settled down.

\section{Outcomes}

1. Primary outcome: The SNOT-20 total score obtained from the Bengali version of the SNOT-20 questionnaire, where, alongside total SNOT-20 score (items 1-20), items are also assigned into six subgroups-psychological domain including fatigue (items 15-20), rhinological (items 1-3, 5, 6), sleep (items 11-13), ear/facial (items 7-10), cough (item 4), and wake up tired (item 14). The scores of each question range from 0 to 5 , according to the severity of the symptom, with 5 being the worst. The score of the SNOT-20 is calculated by summating all the symptom scores, and thus ranges from 0 to $100(20 \times 5=100)$. Additionally, patients can circle those five symptoms that have the highest impact on their impairment. Higher scores on the $0-100$ scale represent lower health-related QOL than lower scores. ${ }^{43}$ Supplementary Files $1 \mathrm{a}$ and $1 \mathrm{~b}$ (available online only) show the English and the Bengali version of the questionnaire respectively.

2. Secondary outcome(s):

(a) The EQ-5D-5L questionnaire, Bengali version (license ID24020), comprises five dimensions: mobility, self-care, usual activities, pain/discomfort, and anxiety or depression. Each dimension has five levels: no problems (1), slight problems (2), moderate problems (3), severe problems (4), and extreme problems (5). Thus, the total raw score ranges 
from 5 to 25. The EuroQol Visual Analog Scale (EQ-VAS) recorded the patient's self-rated health on a vertical VAS of 0 to 100 , where the end points are labeled as: "The worst health you can imagine" and "The best health you can imagine" 44 .

(b) Numeric rating scale (NRS): It measures the severity of sneezing, rhinorrhea, post-nasal drip, facial pain or pressure, and disturbance in sense of smell. ${ }^{45}$

All the outcomes were assessed at baseline, after 2 months, and after 4 months of intervention. All the primary and secondary parameters were self-rated. We have also recorded the (serious) adverse events and intercurrent illnesses.

Sample size: Formal effect size calculation was not possible on account of absence of any earlier study of this explanatory design, i.e., no study to date has tested the efficacy of individualized homeopathic medicines in comparison with placebo in CRS, keeping SNOT-20 scores as the primary outcome, measured over 2 and 4 months. Also, relevant data on SNOT-20 scores were not available for the Bengali and Indian population. A study of 589 patients in Belgium reported the baseline mean \pm standard deviation (SD) of SNOT-20 scores as $41.24 \pm 13.32 .{ }^{46}$ We have assumed the same SD for our study sample and $50 \%$ and $25 \%$ reductions of the mean SNOT-20 scores in the verum and control groups, respectively, over 4 months of intervention. Although the minimal clinically important difference of the SNOT-20 was not determined, a 50\% reduction in the mean score over 4 months of treatment seemed to be a quite reasonable expectation in reflecting the clinical benefits as perceived by the patients in patient-reported outcome. Thus, the assumed means and SDs of the verum and control groups were $20.62 \pm 13.32$ and $10.31 \pm 13.32$ after 4 months of intervention. Effect size (Cohen's $d$ ) was calculated to be 0.774. With this assumed effect size, to detect a significant difference between two independent means (two groups) of SNOT-20 scores after 4 months of intervention through unpaired $t$-test, a study with $2 \times 28$ patients would give the minimum recommendation of $80 \%$ power based on a two-sided significance level of 5\%. Keeping a provision for $10 \%$ attrition, the target sample size becomes 62 (verum: 31 , control: 31).

Randomization: Experimental/verum (IH) or comparator (placebo) was allocated as per a randomization chart generated by using a StatTrek random number generator (https:// stattrek.com/statistics/random-number-generator.aspx; last accessed January 24, 2018). The chart was prepared by an independent third party using restricted six blocks of size 10 $(6 \times 10=60)$ and one block of size 2 , to maintain a $1: 1$ ratio. Thus, equal numbers of patients were randomized to each of the verum and control groups.

Blinding: We adopted the double-blinding method; thus, we achieved allocation concealment by masking of the physicians and the patients throughout the study. Identically coded containers having alike vials of either medicine or placebo were used to maintain concealment. The coded randomization chart was made available to the blinded pharmacist to provide medicines accordingly from the coded vials. Confidentiality was maintained strictly until the end of the trial. Randomization codes were broken at the end of the trial after the dataset was frozen for analysis.

Statistical method: It followed the intention-to-treat (ITT) approach: i.e., every included patient was entered into the final analyses. Missing values were replaced by the series means. Baseline descriptive data (categorical and continuous) were presented in terms of absolute values, percentages, mean, SDs, confidence intervals (CIs), etc., as appropriate. Baseline comparability in socio-demographic features and outcome measures between groups was checked either by chi-square tests (for categorical variables) or by unpaired $t$ test (for continuous variables). Group differences (independent observations) were tested after 2 and 4 months by unpaired $t$-tests. Effect sizes were reported in terms of Cohen's $d$ and were interpreted as small (0.2), medium (0.5), or large (0.8). Dependent observations of continuous outcomes at baseline and at different points of time were compared using repeated-measures analysis of variance (ANOVA). The $p$-values were set at less than 0.05 (two-tailed) as statistically significant. Analysis was performed using Statistical Package for the Social Sciences, version 23.0 (IBM Corp., IBM SPSS Statistics for Windows, Armonk, New York, USA).

Ethical considerations: Irrespective of the assigned codes, adverse events were recorded and treated accordingly as per homeopathic principles. In this project, no new drug was experimented on; nor was any new treatment protocol adopted. Intervention was in strict adherence with classical homeopathy treatment. Prior to enrolment, each patient was provided with a patient information sheet in local vernacular Bengali, detailing the objectives, methods, risks and benefits of participating, and confidentiality issues. Subsequent to that, written informed consent was obtained. Approval was obtained from the IEC prior to initiation of the study. The study was performed under the constant supervision of the IEC. We had prospective registration of the trial protocol in a trial registry, thus making it transparent in conduct and reporting. The protocol conformed to the declaration of Helsinki for ethical conduct of clinical trials involving human participants.

Reporting of adverse events: The investigators had instructed the patients to report any harm, unintended effect, serious adverse event or undue aggravation, either directly in the out-patient departments or over the telephone, during the trial.

Trial reporting: Reporting of the trial was in compliance with the Consolidated Statement for Reporting Trials (CONSORT $)^{47}$ and Reporting Data on Homeopathic Treatment (RedHot) ${ }^{48}$ guidelines (-Supplementary Files $\mathbf{2}$ and $\mathbf{3}$ [available online only]: CONSORT and RedHot checklists).

\section{Results}

Participant flow: According to the previously mentioned inclusion and exclusion criteria, 89 patients suffering from CRS were screened; 27 were excluded due to various reasons (-Fig.1). Sixty-two patients meeting the eligibility criteria 


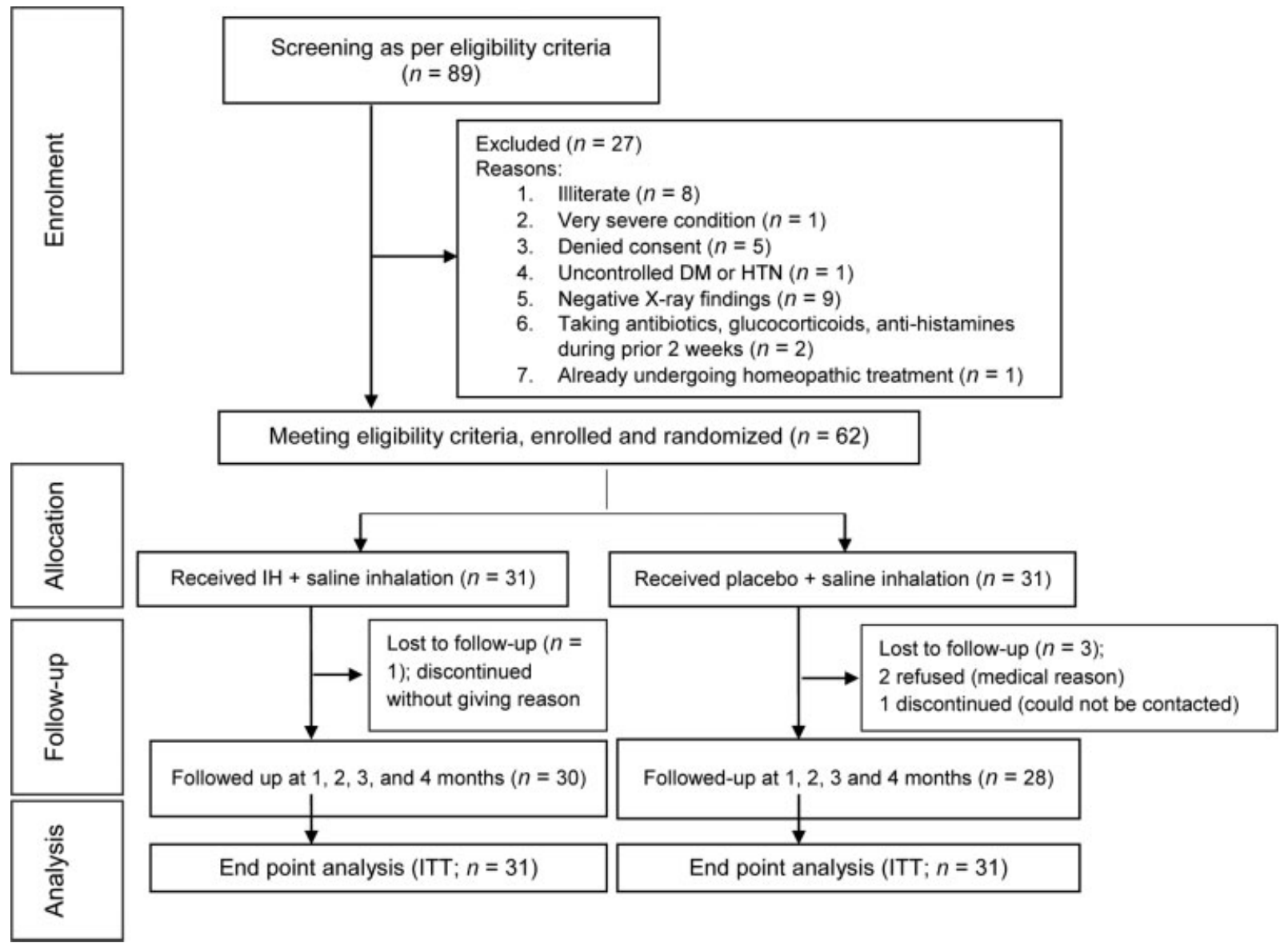

Fig. 1 Study flow diagram.

were enrolled, baseline socio-demographic and outcome data were obtained, and randomized into either verum (IH) or control (identical looking placebo) group, as per the random number chart, by blinded research assistants. After 2 and 4 months of intervention by trained and blinded homeopaths, outcome data were recorded again. During the course of treatment, four patients dropped out from the study (one in the verum group and three in the control group), thus leading to an attrition rate of $6.5 \%$; 58 patients completed the follow-up of 4 months (-Fig. 1: Study flow diagram).

Study duration and follow-up: The total study duration was of 1 year, from March 2018 to February 2019, and each enrolled patient was followed up for 4 months.

Numbers analyzed: All the randomized patients $(n=62)$ entered into the final analysis: that is, 31 in each group.

Baseline data: There were no significant differences (all $p>0.05$, chi-square or unpaired $t$-tests, as appropriate) in the socio-demographic profile between the two groups in terms of age, sex, duration of suffering, type of rhinosinusitis, treatment taken, body mass index, blood pressure, residence, education, employment, or family income status (-Table 1).

Outcomes and estimation:

1. SNOT-20 scores: All the six domains of the SNOT-20 questionnaire-rhinologic, psychological, ear/facial, sleep, cough, wake up tired, and total SNOT-20 questionnaire scores-improved significantly over 2 and 4 months of inter- vention in the verum group (all $p<0.05$, one-way repeatedmeasures ANOVA). On the contrary, in the control group, significant improvements were noted in rhinologic and psychological domains and total SNOT-20 scores; changes in others were non-significant. There were no significant differences at baseline between the two groups in any of the SNOT-20 outcome measures (all $p>0.05$, unpaired $t$-test). Inter-group differences in different SNOT-20 outcomes showed some small but non-significant differences favoring verum over placebo (all $p>0.05$, unpaired $t$-test). After 2 months of intervention, although the achieved mean reduction in total SNOT-20 score was greater in the IH group than for placebo, the difference was statistically non-significant (between-group mean difference: $-1.8,95 \% \mathrm{CI}$ : -9.2 to 5.7, $t$ score at 60 degrees of freedom $[\mathrm{df}]=-0.478$, $p=0.634$, Cohen's $d=0.116$ ). A similar non-significant difference was observed in favor of $\mathrm{IH}$ against placebo after 4 months of intervention (between-group mean difference: $-1.5,95 \% \mathrm{CI}$ : -9.5 to $6.6, t$ score at $\mathrm{df}=60$ was -0.364 , $p=0.717$, Cohen's $d=0.095 ;$ - Table 2).

2. EQ-5D-5L scores: Both the total EQ-5D-5L raw scores and VAS scores were comparable between groups at baseline, with no significant differences between them (both $p>0.05$, unpaired $t$-test). Over 2 and 4 months of intervention, there were significant changes in the verum group in both raw scores and VAS measures ( $p<0.05$, one-way repeated-measures ANOVA). Improvement observed in the control group 
Table 1 Comparison of socio-demographic characteristics between two groups at baseline $(N=62)$

\begin{tabular}{|c|c|c|c|c|c|}
\hline Features & Verum $(n=31)$ & Control $(n=31)$ & $\begin{array}{l}\text { Mean diff. } \pm \text { SE } \\
(95 \% \mathrm{CI})\end{array}$ & $x^{2}$ or $t_{60}$ score & $p$-Value \\
\hline Age (years) ${ }^{a}$ & $33.9 \pm 10.6$ & $33.5 \pm 11.9$ & $0.4 \pm 2.9(-5.4$ to 6.1$)$ & 0.124 & 0.902 \\
\hline \multicolumn{6}{|l|}{ Sex } \\
\hline - Male & $12(38.7)$ & $18(58.1)$ & \multirow[t]{2}{*}{-} & \multirow[t]{2}{*}{2.325} & \multirow[t]{2}{*}{0.127} \\
\hline - Female & $19(61.3)$ & $13(41.9)$ & & & \\
\hline Duration of suffering (years) ${ }^{a}$ & $6.0 \pm 5.0$ & $8.1 \pm 6.8$ & $-2.1 \pm 1.5(-5.2$ to 0.9$)$ & -1.407 & 0.164 \\
\hline \multicolumn{6}{|l|}{ Type $^{b}$} \\
\hline - Maxillary & $18(58.1)$ & $16(51.6)$ & \multirow[t]{5}{*}{-} & \multirow[t]{5}{*}{8.620} & \multirow[t]{5}{*}{0.071} \\
\hline - Frontal & $3(9.7)$ & $4(12.9)$ & & & \\
\hline - Pansinusitis & $6(19.4)$ & $1(3.2)$ & & & \\
\hline - Maxillary + frontal & $2(6.5)$ & $9(29.0)$ & & & \\
\hline - Maxillary + ethmoidal & $2(6.4)$ & $1(3.2)$ & & & \\
\hline \multicolumn{6}{|l|}{ Treatment received $^{\mathrm{b}}$} \\
\hline - None & $11(35.5)$ & $16(51.6)$ & \multirow[t]{3}{*}{-} & \multirow[t]{3}{*}{1.852} & \multirow[t]{3}{*}{0.396} \\
\hline - Allopathy & $16(51.6)$ & $11(35.5)$ & & & \\
\hline - Homeopathy & $4(12.9)$ & $4(12.9)$ & & & \\
\hline Body mass index ${ }^{a}$ & $22.1 \pm 3.2$ & $22.0 \pm 3.3$ & $0.1 \pm 0.8(-1.5$ to 1.8$)$ & 0.158 & 0.875 \\
\hline \multicolumn{6}{|l|}{ Blood pressure $(\mathrm{mm} \mathrm{Hg})^{a}$} \\
\hline - Systolic & $116.9 \pm 14.5$ & $115.5 \pm 15.9$ & $1.4 \pm 3.9(-6.3$ to 9.1$)$ & 0.367 & 0.715 \\
\hline - Diastolic & $80.1 \pm 9.0$ & $77.7 \pm 11.2$ & $2.3 \pm 2.6(-2.8$ to 7.5$)$ & 0.903 & 0.370 \\
\hline \multicolumn{6}{|l|}{ Residence $^{\mathrm{b}}$} \\
\hline - Rural & $8(25.8)$ & $6(19.4)$ & \multirow[t]{3}{*}{-} & \multirow[t]{3}{*}{0.370} & \multirow[t]{3}{*}{0.831} \\
\hline - Semi-urban & $13(41.9)$ & $14(45.2)$ & & & \\
\hline - Urban & $10(32.3)$ & $11(35.5)$ & & & \\
\hline \multicolumn{6}{|l|}{ Educational status $^{\mathrm{b}}$} \\
\hline - 10th std. or less & $21(67.7)$ & $20(64.5)$ & \multirow[t]{2}{*}{-} & \multirow[t]{2}{*}{0.072} & \multirow[t]{2}{*}{0.788} \\
\hline - Higher than 10th std. & $10(32.3)$ & $11(35.5)$ & & & \\
\hline \multicolumn{6}{|l|}{ Employment status $^{\mathrm{b}}$} \\
\hline - Business & $15(48.4)$ & $14(45.2)$ & \multirow[t]{3}{*}{-} & \multirow[t]{3}{*}{4.721} & \multirow[t]{3}{*}{0.094} \\
\hline - Service & $2(6.5)$ & $8(25.8)$ & & & \\
\hline - Dependent & $14(45.2)$ & $9(29.0)$ & & & \\
\hline \multicolumn{6}{|l|}{ Family income status ${ }^{b}$} \\
\hline - Poor & $16(51.6)$ & $15(48.4)$ & \multirow[t]{3}{*}{-} & \multirow[t]{3}{*}{0.418} & \multirow[t]{3}{*}{0.811} \\
\hline - Middle & $10(32.3)$ & $9(29.0)$ & & & \\
\hline - Affluent & $5(16.1)$ & $7(22.6)$ & & & \\
\hline
\end{tabular}

Abbreviations: $\mathrm{Cl}$, confidence interval; SE, standard error.

${ }^{a}$ Continuous data presented as means \pm standard deviations and unpaired $t$-test applied; $t_{60}, t$ score at 60 degrees of freedom.

${ }^{\mathrm{b}}$ Categorical data presented as absolute values (percentages) and chi-square test applied.

Note: $p$-Values less than 0.05 (two-tailed) were considered as statistically significant.

was significant in EQ-5D-5L VASs only ( $p=0.001$, one-way repeated measures ANOVA), but not in raw scores $(p=0.090$, one-way repeated-measures ANOVA). Again, the inter-group differences showed small but non-significant differences favoring verum over placebo (all $p>0.05$, unpaired $t$ test; - Table 3).
3. NRS scores: All the five NRS scores improved significantly from baseline over 2 and 4 months in both the verum and control groups (all $p<0.05$, one-way repeated-measure ANOVA). The two groups were comparable at baseline, without any significant differences between them (all $p>0.05$, unpaired $t$-test). Inter-group differences over 2 
Table 2 Comparison of the SNOT-20 scores at baseline and after 2 and 4 months ( $N=62$; verum: 31 , control: 31$)$

\begin{tabular}{|c|c|c|c|c|c|c|c|}
\hline SNOT-20 domains & $\begin{array}{l}\text { Baseline: } \\
\text { Mean (SD) }\end{array}$ & $\begin{array}{l}\text { After } 2 \text { months: } \\
\text { Mean (SD) }\end{array}$ & $\begin{array}{l}\text { After } 4 \text { months: } \\
\text { Mean (SD) }\end{array}$ & $\begin{array}{l}\text { Wilks' } \\
\text { lambda }\end{array}$ & $\begin{array}{l}\text { Partial eta } \\
\text { squared }\end{array}$ & $F_{2,29}$ & $p$-Value ${ }^{b}$ \\
\hline \multicolumn{8}{|l|}{ Rhinology } \\
\hline - Verum & $14.6(5.6)$ & $10.2(4.3)$ & $9.3(4.5)$ & 0.489 & 0.511 & 15.173 & $<0.001^{\mathrm{c}}$ \\
\hline - Control & $13.5(4.6)$ & $10.3(5.3)$ & $7.9(4.6)$ & 0.489 & 0.511 & 15.171 & $<0.001^{\mathrm{c}}$ \\
\hline Mean group difference (SE) & $1.1(1.3)$ & $-0.1(1.2)$ & $1.4(1.1)$ & & & & \\
\hline $95 \% \mathrm{Cl}$ & -1.5 to 3.7 & -2.5 to 2.3 & -0.8 to 3.7 & & & & \\
\hline$t_{60}$ & 0.852 & -0.084 & 1.265 & & & & \\
\hline$p$-Value ${ }^{a}$ & 0.397 & 0.933 & 0.211 & & & & \\
\hline Cohen's d & - & 0.021 & 0.321 & & & & \\
\hline \multicolumn{8}{|l|}{ Ear/Facial } \\
\hline - Verum & $8.1(4.1)$ & $6.7(3.4)$ & $6.3(3.7)$ & 0.723 & 0.277 & 0.552 & $0.009^{c}$ \\
\hline - Control & $7.2(5.2)$ & $6.2(3.5)$ & $6.1(4.0)$ & 0.944 & 0.056 & 0.858 & 0.434 \\
\hline Mean group difference (SE) & $0.9(1.2)$ & $0.5(0.9)$ & $0.2(1.0)$ & & & & \\
\hline $95 \% \mathrm{Cl}$ & -1.5 to 3.3 & -1.2 to 2.3 & -1.7 to 2.2 & & & & \\
\hline$t_{60}$ & 0.735 & 0.602 & 0.224 & & & & \\
\hline$p$-Value ${ }^{a}$ & 0.465 & 0.550 & 0.824 & & & & \\
\hline Cohen's $d$ & - & 0.157 & 0.057 & & & & \\
\hline \multicolumn{8}{|l|}{ Sleep } \\
\hline - Verum & $7.1(4.7)$ & $4.5(4.0)$ & $4.7(4.1)$ & 0.644 & 0.356 & 8.004 & $0.002^{c}$ \\
\hline - Control & $6.7(5.1)$ & $5.4(4.1)$ & $5.7(4.6)$ & 0.866 & 0.134 & 2.249 & 0.124 \\
\hline Mean group difference (SE) & $0.4(1.2)$ & $-0.9(1.0)$ & $-1.0(1.1)$ & & & & \\
\hline $95 \% \mathrm{Cl}$ & -2.2 to 2.8 & -3.0 to 1.1 & -3.2 to 1.2 & & & & \\
\hline$t_{60}$ & 0.258 & -0.909 & -0.904 & & & & \\
\hline$p$-Value ${ }^{a}$ & 0.798 & 0.367 & 0.369 & & & & \\
\hline Cohen's d & - & 0.233 & 0.230 & & & & \\
\hline \multicolumn{8}{|l|}{ Psychological } \\
\hline - Verum & $18.0(7.2)$ & $12.1(6.9)$ & $10.2(6.2)$ & 0.494 & 0.506 & 14.870 & $<0.001^{\mathrm{c}}$ \\
\hline - Control & $16.3(6.7)$ & $12.3(7.0)$ & $11.8(6.6)$ & 0.696 & 0.304 & 6.341 & $0.005^{c}$ \\
\hline Mean group difference (SE) & $1.7(1.8)$ & $-0.2(1.7)$ & $-1.6(1.6)$ & & & & \\
\hline $95 \% \mathrm{Cl}$ & -1.9 to 5.1 & -3.7 to 3.3 & -4.8 to 1.6 & & & & \\
\hline$t_{60}$ & 0.929 & -0.136 & -0.981 & & & & \\
\hline$p$-Value ${ }^{a}$ & 0.357 & 0.893 & 0.331 & & & & \\
\hline Cohen's $d$ & - & 0.035 & 0.249 & & & & \\
\hline \multicolumn{8}{|l|}{ Cough } \\
\hline - Verum & $2.0(1.6)$ & $1.1(1.2)$ & $1.3(1.1)$ & 0.748 & 0.252 & 4.884 & $0.015^{c}$ \\
\hline - Control & $2.2(1.6)$ & $1.8(1.4)$ & $1.5(1.4)$ & 0.864 & 0.136 & 2.277 & 0.121 \\
\hline Mean group difference (SE) & $-0.2(0.4)$ & $-0.7(0.3)$ & $-0.2(0.3)$ & & & & \\
\hline $95 \% \mathrm{Cl}$ & -0.9 to 0.7 & -1.3 to 0.02 & -0.9 to 0.4 & & & & \\
\hline$t_{60}$ & -0.324 & -1.937 & -0.740 & & & & \\
\hline$p$-Value ${ }^{a}$ & 0.747 & 0.057 & 0.462 & & & & \\
\hline Cohen's d & - & 0.489 & 0.189 & & & & \\
\hline \multicolumn{8}{|l|}{ Wake up tired } \\
\hline - Verum & $2.9(1.8)$ & $2.3(1.4)$ & $1.9(1.5)$ & 0.766 & 0.234 & 4.430 & $0.021^{c}$ \\
\hline - Control & $2.9(1.7)$ & $2.7(1.5)$ & $2.2(1.5)$ & 0.850 & 0.150 & 2.552 & 0.095 \\
\hline Mean group difference (SE) & $-0.1(0.4)$ & $-0.4(0.4)$ & $-0.3(0.4)$ & & & & \\
\hline
\end{tabular}


Table 2 (Continued)

\begin{tabular}{|c|c|c|c|c|c|c|c|}
\hline SNOT-20 domains & $\begin{array}{l}\text { Baseline: } \\
\text { Mean (SD) }\end{array}$ & $\begin{array}{l}\text { After } 2 \text { months: } \\
\text { Mean (SD) }\end{array}$ & $\begin{array}{l}\text { After } 4 \text { months: } \\
\text { Mean (SD) }\end{array}$ & $\begin{array}{l}\text { Wilks' } \\
\text { lambda }\end{array}$ & $\begin{array}{l}\text { Partial eta } \\
\text { squared }\end{array}$ & $F_{2,29}$ & $p$-Value ${ }^{b}$ \\
\hline $95 \% \mathrm{Cl}$ & -1.0 to 0.8 & -1.1 to 0.4 & -1.0 to 0.4 & & & & \\
\hline$t_{60}$ & -0.145 & -1.008 & -0.794 & & & & \\
\hline$p$-Value ${ }^{a}$ & 0.886 & 0.317 & 0.430 & & & & \\
\hline Cohen's $d$ & - & 0.256 & 0.203 & & & & \\
\hline \multicolumn{8}{|l|}{ Total score } \\
\hline - Verum & $52.6(16.5)$ & $36.9(13.4)$ & $33.8(13.2)$ & 0.371 & 0.629 & 24.604 & $<0.001^{\mathrm{C}}$ \\
\hline - Control & $48.9(17.2)$ & $38.7(15.8)$ & $35.3(18.0)$ & 0.575 & 0.425 & 10.716 & $<0.001^{c}$ \\
\hline Mean group difference (SE) & $3.7(4.3)$ & $-1.8(3.7)$ & $-1.5(4.0)$ & & & & \\
\hline $95 \% \mathrm{Cl}$ & -4.8 to 12.3 & -9.2 to 5.7 & -9.5 to 6.5 & & & & \\
\hline$t_{60}$ & 0.876 & -0.478 & -0.364 & & & & \\
\hline$p$-Value ${ }^{a}$ & 0.384 & 0.634 & 0.717 & & & & \\
\hline Cohen's $d$ & - & 0.116 & 0.095 & & & & \\
\hline
\end{tabular}

Abbreviations: ANOVA, analysis of variance; $\mathrm{Cl}$, confidence interval; SD, standard deviation; SE, standard error; SNOT, sino-nasal outcome test; $t_{60}, t$ score at 60 degrees of freedom.

anpaired $t$-tests.

${ }^{\mathrm{b}}$ One-way repeated-measures analysis of variance.

${ }^{c} p<0.05$ considered as statistically significant.

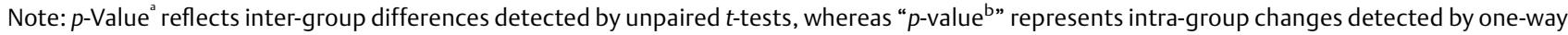
repeated-measures ANOVA. Lambda is the measure of the percentage variance in dependent variables not explained by differences in levels of the independent variable. The null hypothesis is rejected when Wilks' lambda is close to zero; Eta is the measure of effect size for use in ANOVA and analogous to $R^{2}$ from multiple linear regressions.

Table 3 Comparison of the EQ-5D-5L scores at baseline and after 2 and 4 months ( $N=62$; verum: 31 , control: 31$)$

\begin{tabular}{|c|c|c|c|c|c|c|c|}
\hline EQ-5D-5L scores & $\begin{array}{l}\text { Baseline: } \\
\text { Mean (SD) }\end{array}$ & $\begin{array}{l}\text { After } 2 \text { months: } \\
\text { Mean (SD) }\end{array}$ & $\begin{array}{l}\text { After } 4 \text { months: } \\
\text { Mean (SD) }\end{array}$ & $\begin{array}{l}\text { Wilks' } \\
\text { lambda }\end{array}$ & $\begin{array}{l}\text { Partial eta } \\
\text { squared }\end{array}$ & $F_{2,29}$ & $p$-Value ${ }^{\mathrm{b}}$ \\
\hline \multicolumn{8}{|l|}{ Total raw scores } \\
\hline - Verum & $6.5(4.0)$ & $5.4(3.7)$ & $4.2(3.3)$ & 0.757 & 0.243 & 4.655 & $0.018^{c}$ \\
\hline - Control & $6.3(3.1)$ & $5.4(3.6)$ & $5.0(3.2)$ & 0.847 & 0.153 & 2.616 & 0.090 \\
\hline Mean group difference (SE) & $0.2(0.9)$ & $-0.01(0.9)$ & $-0.8(0.8)$ & & & & \\
\hline $95 \% \mathrm{Cl}$ & -1.6 to 2.0 & -1.9 to 1.9 & -2.5 to 0.8 & & & & \\
\hline$t_{60}$ & 0.201 & -0.01 & -0.991 & & & & \\
\hline$p$-Value ${ }^{a}$ & 0.842 & 0.992 & 0.326 & & & & \\
\hline Cohen's d & - & 0 & 0.246 & & & & \\
\hline \multicolumn{8}{|l|}{ VAS scores } \\
\hline - Verum & $58.4(14.6)$ & $68.5(13.2)$ & $71.7(14.6)$ & 0.508 & 0.492 & 14.015 & $<0.001^{\mathrm{c}}$ \\
\hline - Control & $57.7(15.0)$ & $65.7(17.3)$ & $71.5(15.8)$ & 0.598 & 0.402 & 9.761 & $0.001^{c}$ \\
\hline Mean group difference (SE) & $0.6(3.8)$ & $2.8(3.9)$ & $0.2(3.9)$ & & & & \\
\hline $95 \% \mathrm{Cl}$ & -6.9 to 8.2 & -5.0 to 10.7 & -7.5 to 7.9 & & & & \\
\hline$t_{60}$ & 0.171 & 0.725 & 0.060 & & & & \\
\hline$p$-Value ${ }^{a}$ & 0.864 & 0.471 & 0.952 & & & & \\
\hline Cohen's $d$ & - & 0.182 & 0.013 & & & & \\
\hline
\end{tabular}

Abbreviations: ANOVA, analysis of variance; $\mathrm{Cl}$, confidence interval; SD, standard deviation; SE, standard error.

anpaired $t$-tests.

${ }^{\mathrm{b}}$ One-way repeated-measures analysis of variance.

${ }^{c} p<0.05$ considered as statistically significant.

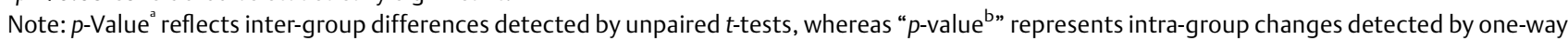
repeated measure ANOVA. Lambda is the measure of the percentage variance in dependent variables not explained by differences in levels of the independent variable. The null hypothesis is rejected when Wilks' lambda is close to zero; Eta is the measure of effect size for use in ANOVA and analogous to $R^{2}$ from multiple linear regressions. 
Table 4 Comparison of the NRS (0-10) scores at baseline and after 2 and 4 months ( $N=62$; verum: 31 , control: 31$)$

\begin{tabular}{|c|c|c|c|c|c|c|c|}
\hline NRS & $\begin{array}{l}\text { Baseline: } \\
\text { Mean (SD) }\end{array}$ & $\begin{array}{l}\text { After } 2 \text { months: } \\
\text { Mean (SD) }\end{array}$ & $\begin{array}{l}\text { After } 4 \text { months: } \\
\text { Mean (SD) }\end{array}$ & $\begin{array}{l}\text { Wilks' } \\
\text { lambda }\end{array}$ & $\begin{array}{l}\text { Partial eta } \\
\text { squared }\end{array}$ & $F_{2,29}$ & $p$-Value ${ }^{\mathrm{b}}$ \\
\hline \multicolumn{8}{|l|}{ Sneezing } \\
\hline - Verum & $6.9(2.9)$ & $4.7(3.0)$ & $3.9(2.9)$ & 0.440 & 0.560 & 18.491 & $<0.001^{c}$ \\
\hline - Control & $6.8(2.6)$ & $4.5(2.9)$ & $4.3(2.6)$ & 0.573 & 0.427 & 10.806 & $<0.001^{\mathrm{c}}$ \\
\hline Mean group difference (SE) & $0.1(0.7)$ & $0.1(0.8)$ & $-0.4(0.7)$ & & & & \\
\hline $95 \% \mathrm{Cl}$ & -1.3 to 1.5 & -1.4 to 1.6 & -1.8 to 1.0 & & & & \\
\hline$t_{60}$ & 0.137 & 0.158 & -0.570 & & & & \\
\hline$p$-Value ${ }^{a}$ & 0.892 & 0.875 & 0.571 & & & & \\
\hline Cohen's $d$ & - & 0.068 & 0.145 & & & & \\
\hline \multicolumn{8}{|l|}{ Rhinorrhea } \\
\hline - Verum & $6.5(3.3)$ & $4.1(3.3)$ & $3.2(3.0)$ & 0.490 & 0.510 & 15.084 & $<0.001^{\mathrm{c}}$ \\
\hline - Control & $6.0(2.9)$ & $4.4(3.0)$ & $3.5(2.5)$ & 0.696 & 0.304 & 6.347 & $0.005^{c}$ \\
\hline Mean group difference (SE) & $0.5(0.8)$ & $-0.3(0.8)$ & $-0.3(0.7)$ & & & & \\
\hline $95 \% \mathrm{Cl}$ & -1.1 to 2.1 & -1.9 to 1.3 & -1.7 to 1.1 & & & & \\
\hline$t_{60}$ & 0.616 & -0.390 & -0.452 & & & & \\
\hline$p$-Value ${ }^{a}$ & 0.540 & 0.698 & 0.653 & & & & \\
\hline Cohen's $d$ & - & 0.095 & 0.109 & & & & \\
\hline \multicolumn{8}{|l|}{ Post-nasal drip } \\
\hline - Verum & $6.5(3.2)$ & $4.4(3.2)$ & $3.4(2.8)$ & 0.492 & 0.508 & 14.992 & $<0.001^{\mathrm{c}}$ \\
\hline - Control & $6.0(2.6)$ & $3.3(2.6)$ & $3.3(2.5)$ & 0.491 & 0.509 & 15.054 & $<0.001^{\mathrm{c}}$ \\
\hline Mean group difference (SE) & $0.6(0.7)$ & $1.1(0.7)$ & $0.1(0.7)$ & & & & \\
\hline $95 \% \mathrm{Cl}$ & -0.9 to 2.0 & -0.4 to 2.6 & -1.2 to 1.5 & & & & \\
\hline$t_{60}$ & 0.797 & 1.450 & 0.184 & & & & \\
\hline$p$-Value ${ }^{a}$ & 0.428 & 0.152 & 0.855 & & & & \\
\hline Cohen's $d$ & - & 0.377 & 0.038 & & & & \\
\hline \multicolumn{8}{|l|}{ Facial pain/pressure } \\
\hline - Verum & $6.7(3.3)$ & $4.1(3.6)$ & $3.3(3.2)$ & 0.553 & 0.447 & 11.737 & $<0.001^{c}$ \\
\hline - Control & $5.0(3.3)$ & $2.6(2.4)$ & $2.4(2.3)$ & 0.547 & 0.453 & 12.019 & $<0.001^{\mathrm{c}}$ \\
\hline Mean group difference (SE) & $1.7(0.8)$ & $1.5(0.8)$ & $0.9(0.7)$ & & & & \\
\hline $95 \% \mathrm{Cl}$ & -0.0003 to 3.3 & -0.001 to 3.1 & -0.5 to 2.3 & & & & \\
\hline$t_{60}$ & 2.000 & 1.998 & 1.327 & & & & \\
\hline$p$-Value ${ }^{a}$ & 0.050 & 0.050 & 0.189 & & & & \\
\hline Cohen's $d$ & - & 0.490 & 0.323 & & & & \\
\hline \multicolumn{8}{|l|}{ Disturbance in sense of smell } \\
\hline - Verum & $5.1(4.4)$ & $3.5(3.9)$ & $3.0(3.5)$ & 0.731 & 0.269 & 5.329 & $0.011^{\mathrm{c}}$ \\
\hline - Control & $5.0(3.2)$ & $3.4(3.1)$ & $3.2(2.9)$ & 0.699 & 0.301 & 6.246 & $0.006^{c}$ \\
\hline Mean group difference (SE) & $0.2(1.0)$ & $0.1(0.9)$ & $-0.2(0.8)$ & & & & \\
\hline $95 \% \mathrm{Cl}$ & -1.8 to 2.1 & -1.7 to 1.9 & -1.9 to 1.4 & & & & \\
\hline$t_{60}$ & 0.165 & 0.115 & -0.291 & & & & \\
\hline$p$-Value ${ }^{a}$ & 0.869 & 0.909 & 0.772 & & & & \\
\hline Cohen's $d$ & - & 0.028 & 0.062 & & & & \\
\hline
\end{tabular}

Abbreviations: ANOVA, analysis of variance; $\mathrm{Cl}$, confidence interval; NRS, numeric rating scale; SD, standard deviation; SE, standard error.

anpaired $t$-tests.

${ }^{\mathrm{b} O n e-w a y ~ r e p e a t e d-m e a s u r e s ~ a n a l y s i s ~ o f ~ v a r i a n c e . ~}$

${ }^{c} p<0.05$ considered as statistically significant.

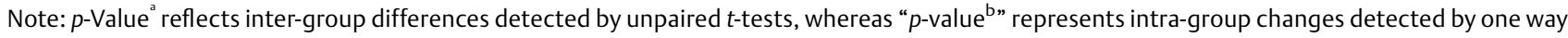
repeated measure ANOVA; Lambda is the measure of the percentage variance in dependent variables not explained by differences in levels of the independent variable. The null hypothesis is rejected when Wilks' lambda is close to zero; Eta is the measure of effect size for use in ANOVA and analogous to $R^{2}$ from multiple linear regressions. 
and 4 months showed small but non-significant differences (all $p>0.05$, unpaired $t$-test), with small to medium effect sizes in favor of verum ( - Table 4 ).

Medicines used: Overall, 27 different medicines were prescribed in $30 \mathrm{cH}, 200 \mathrm{cH}$, and $1000 \mathrm{cH}$ potencies. The most frequently indicated medicines were Calcarea carbonica, Lycopodium clavatum, and Sulphur ( $n=6$ each; 9.7\% each), Natrum muriaticum and Pulsatilla nigricans ( $n=5$ each; $8.1 \%$ each), and Arsenicum album $(n=4 ; 6.5 \%)$. The most frequently indicated medicines in the verum group were Lycopodium clavatum ( $n=5 ; 16.1 \%)$, Pulsatilla nigricans $(n=4 ; 12.9 \%)$, Calcarea carbonicum and Natrum muriaticum ( $n=3$ each; $9.7 \%$ each). In the control group, the most frequently indicated medicines were Sulphur ( $n=4 ; 12.9 \%)$, Arsenicum album and Calcarea carbonica ( $n=3$ each; $9.7 \%$ each), and Allium cepa, Natrum muriaticum, Natrum sulphuricum, Nux vomica and Phosphorus ( $n=2$ each; $6.5 \%$ each) (-Table 5 ). For details, see
- Supplementary File 4 (available online only): Indications of the prescribed medicines.

Adverse events: No harms, unintended effects, homeopathic aggravations or any serious adverse events were reported from either group of patients during the intervention period. Two adverse events were reported in the verum group-one case of non-specific pruritic eruptions on both the lower limbs, and a case of fever. The former was prescribed Echinacea angustifolia tincture as external application, and that had healed the case. The latter individual took over-the-counter paracetamol, $650 \mathrm{mg}$ two tablets 6 hourly, and recovered. Two adverse events of pruritic eruptions on the body occurred in the control group: they were prescribed Calendula officinalis tincture and Echinacea angustifolia tincture respectively as external application. One patient in the control group sustained an injury during the study; he was prescribed Arnica montana $30 \mathrm{cH}$, three doses 8 hourly, and that was sufficient to manage the condition.

Table 5 Alphabetical list of medicines prescribed in the two groups at baseline

\begin{tabular}{|c|c|c|c|c|c|}
\hline Name of the medicines & $\begin{array}{l}\text { Total }(N=62) \\
n(\%)\end{array}$ & $\begin{array}{l}\text { Verum }(n=31) \\
n(\%)\end{array}$ & $\begin{array}{l}\text { Control }(n=31) \\
n(\%)\end{array}$ & Chi-square & $p$-Value ${ }^{a}$ \\
\hline 1. Allium сера & $2(3.2)$ & - & $2(6.5)$ & - & - \\
\hline 2. Arsenicum album & $4(6.5)$ & $1(3.2)$ & $3(9.7)$ & 0.267 & 0.605 \\
\hline 3. Bromium & $1(1.6)$ & $1(3.2)$ & - & - & - \\
\hline 4. Calcarea carbonica & $6(9.7)$ & $3(9.7)$ & $3(9.7)$ & 0.185 & 0.668 \\
\hline 5. Calcarea phosphorica & $1(1.6)$ & $1(3.2)$ & - & - & - \\
\hline 6. Carbo vegetabilis & $1(1.6)$ & $1(3.2)$ & - & - & - \\
\hline 7. Carcinosinum & $2(3.2)$ & $2(6.5)$ & - & - & - \\
\hline 8. Causticum & $2(3.2)$ & $2(6.5)$ & - & - & - \\
\hline 9. Hepar sulphuris & $1(1.6)$ & - & $1(3.2)$ & - & - \\
\hline 10. Kali muriaticum & $1(1.6)$ & - & $1(3.2)$ & - & - \\
\hline 11. Kali sulphuricum & $1(1.6)$ & - & $1(3.2)$ & - & - \\
\hline 12. Lachesis mutus & $1(1.6)$ & $1(3.2)$ & - & - & - \\
\hline 13. Lycopodium clavatum & $6(9.7)$ & $5(16.1)$ & $1(3.2)$ & 1.661 & 0.198 \\
\hline 14. Medorrhinum & $1(1.6)$ & $1(3.2)$ & - & - & - \\
\hline 15. Mercurius solubilis & $1(1.6)$ & - & $1(3.2)$ & - & - \\
\hline 16. Natrum muriaticum & $5(8.1)$ & $3(9.7)$ & $2(6.5)$ & 0.000 & 1.000 \\
\hline 17. Natrum sulphuricum & $2(3.2)$ & - & $2(6.5)$ & - & - \\
\hline 18. Nitric acid & $1(1.6)$ & $1(3.2)$ & - & - & - \\
\hline 19. Nux vomica & $3(4.8)$ & $1(3.2)$ & $2(6.5)$ & 0.000 & 1.000 \\
\hline 20. Phosphorus & $2(3.2)$ & - & $2(6.5)$ & - & - \\
\hline 21. Pulsatilla nigricans & $5(8.1)$ & $4(12.9)$ & $1(3.2)$ & 0.870 & 0.351 \\
\hline 22. Rhus toxicodendron & $1(1.6)$ & - & $1(3.2)$ & - & - \\
\hline 23. Sepia succus & $3(4.8)$ & $2(6.5)$ & $1(3.2)$ & 0.000 & 1.000 \\
\hline 24. Staphysagria & $1(1.6)$ & - & $1(3.2)$ & - & - \\
\hline 25. Sulphur & $6(9.7)$ & $2(6.5)$ & $4(12.9)$ & 0.185 & 0.668 \\
\hline 26. Tarantula hispanica & $1(1.6)$ & - & $1(3.2)$ & - & - \\
\hline 27. Tuberculinum bovinum & $1(1.6)$ & - & $1(3.2)$ & - & - \\
\hline
\end{tabular}

apearson's chi-square test. 


\section{Discussion}

In this double-blind RCT conducted on 62 patients suffering from CRS, a small though non-significant direction of effect was observed favoring IH over placebo in both primary and secondary outcome measures. Larger and rigorous trials and independent replications are warranted.

The trial design was gold standard-that is an RCT. The enrolment into the trial was prospective; i.e., the protocol was declared before the enrollment of the first patient. All the collected data in the form of case records and scales were converted into an analyzable and reproducible master chart (soft copy, Microsoft Excel spreadsheet) and underwent statistical analysis subsequently. Validated outcome measures, such as SNOT-20, EQ-5D-5L and NRS scores, were used to assess the severity of symptoms and QOL. Missing values were replaced by the series means; thus an intention-totreat approach was adopted, where all the enrolled patients entered into the final analysis. The treatment was given adhering to the classical principles of homeopathy, based on individualization and totality of symptoms. Within a span of 8 months, we screened 89 patients, from whom 62 could be enrolled, thus reflecting the possibility of larger trials in the same setting in the future. The inclusion/screening rate of $(62 / 89) \times 100=69.7 \%$ and retention rate of $(58 /$ $62) \times 100=93.5 \%$ were also promising. We developed the Bengali version of SNOT-20 through a standardized forwardbackward translation method and tested its psychometric properties and cross-cultural adaptability during April to September 2017 prior to use in the trial, and found it to be satisfactory; being beyond the scope of this article it will be reported elsewhere. The developed Bengali SNOT-20 questionnaire was also used in an open-label, single-arm trial testing the effects of individualized homeopathic medicines in sino-nasal symptoms in patients suffering from nasal polyps, with promising results. ${ }^{49}$

The duration of the study might be considered as inadequate, especially in a chronic condition such as CRS that shows seasonal variations and intermittent exacerbations. A longer follow-up period might have been more appropriate; however, the feasibility and ethical issues of conducting an explanatory trial with longer duration of follow-ups should be kept in mind. While treating a chronic condition, often a series of homeopathic remedies might be required in succession instead of a single medicine to initiate a favorable response. ${ }^{50}$ So a timeline of only 4 months could be considered as inadequate. The small sample size would have inflated the chances of type II error; therefore, the chance of false-negative results could not be ruled out. All the patient outcome measures in this study were subjective and self-administered, so they were amenable to bias; however, that potential deficiency would have been balanced by the randomization and baseline comparability. The rigorous case taking and patient interaction might cause additional subjective bias but any such effects would have been the same for each group.

There have been few studies assessing the possible effects of homeopathic medicines in cases of sinusitis; only a few studies have been performed with IH in single-arm, open- label design ${ }^{38-41}$; in the remainder (majority) of the studies, medicines were either pre-defined single or complex medicines. The study by Nayak et al ${ }^{38}$ was an open-label, singlearm study conducted on 628 patients suffering from chronic sinusitis. Seventeen homeopathic medicines were shortlisted based on repertorization of the disease symptoms. The scale used as the outcome measure was a chronic sinusitis assessment score that was developed by the CCRH. Positive results were detected and the need of a controlled trial was mentioned. In comparison with that study ours was of gold standard design, evaluating the efficacy of IH in comparison with placebo in treatment of CRS. Second, unlike Nayak et $\mathrm{al}^{38}$ we used all validated outcome measures-SNOT-20, EQ-5D-5L, and NRS scoresjudging both symptom severity and QOL. A few of our used medicines were similar to those of Nayak et al: ${ }^{38}$ for example, Calcarea carbonica, Lycopodium clavatum, Pulsatilla nigricans, Arsenicum album, Phosphorus and Nux vomica. However, some of their most frequently prescribed medicines, including Silicea terra, Kali iodatum and Kali bichromicum, were not prescribed in our trial. The probable reason is the differences in approach in selection of medicines. Shortlisting of remedies on the basis of repertorization, encompassing all the available disease symptoms and ignoring general characteristic symptoms (physical or mental), is not strictly in accordance with the classical principles of homeopathy or repertorization. Also, unlike Nayak et al, ${ }^{38}$ we refrained from arbitrary categorization of the outcomes from "marked improvement" to "worse".

Our trial sample size was much smaller than that in the studies by Nayak et $\mathrm{al}^{38}$ or by Manchanda ${ }^{42}$; however, increasing the sample size further was not possible owing to time constraints of completing the project and submitting the dissertation to the university. Unlike Manchanda, ${ }^{42}$ we refrained from using a 14-day run-in period prior to enrollment and 6 months' of follow-up, as these seemed to be not feasible and ethically objectionable. Also unlike Manchanda, ${ }^{42}$ we did not use the effect size estimated from an observational study (usually over-estimated) in calculation of sample size for the RCT.

Two other studies, by Sharma et $\mathrm{al}^{39}$ and Ramteke et al, ${ }^{40}$ were similar to that of Nayak et al in terms of weak study design, use of a non-validated outcome measure, pre-defined medicines, restriction to analysis of the protocol-compliant sample, and loose interpretation of results; however, the sample size attained by Sharma et al $(n=950)$ was much higher than that obtained by Ramteke et al $(n=97)$. Thus, both in terms of study design and statistical analysis (restricted to protocol-compliant sample only), all these three studies of the CCRH were less rigorous than this current trial.

The observational study by Witt et $\mathrm{al}^{41}$ was superior to the CCRH studies in terms of the longer duration of the follow-up of 8 years, use of validated outcome measures (symptom severity NRS, SF-36), repeat measures and ITT analysis, as well as prescription of $\mathrm{IH}$, but it suffered from obvious design constraints. That study showed strongest improvements in the severity of complaints in the first 3 months that generally continued during the first 24 months and persisted for 
another 6 years, with large reductions in the use of conventional medicines and health care services. The physicians' assessments of the severity change tended to be more positive than patients' own assessments. This was the reason we were sceptical about having physician-assessed measures as outcomes, and we opted for patient-reported outcomes.

In the older homeopathy literature, successful treatment of 33 consecutive cases of sinusitis with various $\mathrm{IH}$ in potencies higher than $30 \mathrm{cH}$ has also been noted, where one-third reported relief within 1 month. ${ }^{51} \mathrm{Dr}$. Vithoulkas has mentioned another case of sinusitis with somnambulism, and treated successfully with a series of homeopathic medicines such as Cocculus indicus, Nux vomica and Calcarea carbonica. $^{52}$ In another study by Peters et al, ${ }^{53}$ two approaches were suggested-"pathological" and "general or constitutional". The former prescribing technique has similarity with the CCRH studies, ${ }^{38-40}$ whilst the latter is similar to that of Witt et $\mathrm{al}^{41}$ and ours.

Nasal endoscopy (NE) is of great use in direct visualization of the nasal cavity mucosa and identification of the presence of any pathology or polyps. On the other hand, given its resolution of the regional anatomy and mucosa, computed tomography (CT) is an optimal modality and the gold standard to provide the diagnosis of CRS and an anatomical roadmap for the surgeon performing the functional endoscopic sinus surgery. Neither CT nor NE was possible in our setting and we had to rely on X-ray reporting of paranasal sinuses (occipito-mento view) for the diagnosis of CRS and confirmed by the blinded ENT surgeon. Hence, CT and NE must be used together in the diagnosis of $\mathrm{CRS}^{54}$ in future trials. Subject to standardized translation into Bengali, psychometric validation and cross-cultural adaptation, the updated SNOT-22 questionnaire may also be used in the future. All the indicated homeopathic medicines in this study were prescribed in centesimal potencies; 50 millesimal potencies were not used, and that can be one promising option to be evaluated in future trials.

\section{Conclusion}

Overall, the trial remained inconclusive owing to the small and non-significant trend of effect revealed, favoring homeopathy over placebo. Robust multicentric trials with large sample size and independent replications should be undertaken to arrive at a definite conclusion regarding the efficacy of IH medicines in the treatment of CRS.

\section{Highlights}

- A double-blind, randomized, placebo-controlled, clinical trial was conducted at the National Institute of Homoeopathy, West Bengal, India, in 62 patients suffering from chronic rhinosinusitis.

- Though improvements were greater in the homeopathy group than in the placebo group, with small to medium effect sizes, the group differences were statistically nonsignificant, thus rendering the trial inconclusive.
- Calcarea carbonica, Lycopodium clavatum, Sulphur, Natrum muriaticum and Pulsatilla nigricans were the most frequently prescribed medicines.

\section{Supplementary File}

Supplementary File 1 Sino-nasal outcome test Supplementary File 2 CONSORT 2010 checklist of information to include when reporting a randomized trial Supplementary File 3 RedHot checklist of information to include when reporting randomized trials of homeopathy Supplementary File 4 Indications of the prescribed medicines

\section{Authors' Contributions}

P.M., C.N., A.C. and T.K.P. contributed to the study concept, literature search, conducting the clinical study, data acquisition, data interpretation, and drafting the manuscript. B.G., S.S., K.B., S.R. and M.P. contributed to conducting the clinical study and data acquisition. S.S.A. and A.B. contributed to data management. A.N., M.K. and S.S. contributed to study design, data interpretation, statistical analysis, and drafting the manuscript. All the authors reviewed and approved the final manuscript.

\section{Note}

The trial was performed as part of the postgraduate thesis of the corresponding author under the guidance of C.N. A.C. is the Head of the Department of Materia Medica, National Institute of Homoeopathy. Authors B.G., S.S., K.B., S.R. and M.P. are postgraduate trainees at the National Institute of Homoeopathy. A.N. is a former postgraduate trainee at the National Institute of Homoeopathy. S.A. and A.B. are interns at Mahesh Bhattacharyya Homoeopathic Medical College \& Hospital. MK and S.S. are independent researchers.

\section{Funding}

None. The institution had no role to play in the analysis of the study results or the preparation of the paper.

\section{Conflict of Interest}

None declared.

\section{Acknowledgment}

The authors are grateful to institutional heads, both academic and hospital, for allowing us to conduct the trial. We sincerely thank the fellow postgraduate trainees, staff, pharmacists and the patients for their sincere participation in the study.

\section{References}

1 Orlandi RR, Kingdom TT, Hwang PH, et al. International consensus statement on allergy and rhinology: rhinosinusitis. Int Forum Allergy Rhinol 2016;6:S22-S209 
2 Suh JD, Kennedy DW. Treatment options for chronic rhinosinusitis. Proc Am Thorac Soc 2011;8:132-140

3 Lohiya SS, Patel SV, Pawde AM, Bokare BD, Sakhare PT. Comparative study of diagnostic nasal endoscopy and CT paranasal sinuses in diagnosing chronic rhinosinusitis. Indian J Otolaryngol Head Neck Surg 2016;68:224-229

4 Rosenfeld RM, Piccirillo JF, Chandrasekhar SS, et al. Clinical practice guideline (update): adult sinusitis. Otolaryngol Head Neck Surg 2015;152:S1-S39

5 Cox DR, Ashby S, DeConde AS, et al. Dyad of pain and depression in chronic rhinosinusitis. Int Forum Allergy Rhinol 2016; 6:308-314

6 Alt JA, Smith TL, Schlosser RJ, Mace JC, Soler ZM. Sleep and quality of life improvements after endoscopic sinus surgery in patients with chronic rhinosinusitis. Int Forum Allergy Rhinol 2014; 4:693-701

7 Alam ES, Musselman DL, Chyou D, et al. Somatization, depression, and anxiety disorders in a rhinology practice. Am J Rhinol Allergy 2019;33:470-477

8 Kariyawasam HH, Scadding GK. Chronic rhinosinusitis: therapeutic efficacy of anti-inflammatory and antibiotic approaches. Allergy Asthma Immunol Res 2011;3:226-235

9 DeConde AS, Soler ZM. Chronic rhinosinusitis: epidemiology and burden of disease. Am J Rhinol Allergy 2016;30:134-139

10 Meltzer EO, Bukstein DA. The economic impact of allergic rhinitis and current guidelines for treatment. Ann Allergy Asthma Immunol 2011;106:S12-S16

11 Bhargava SK, Peethambaran K. Pharmacotherapy of chronic rhinosinusitis with a focus on clarithromycin: an expert opinion. J Assoc Physicians India 2018;66:74-79

12 Young LC, Stow NW, Zhou L, Douglas RG. Efficacy of medical therapy in treatment of chronic rhinosinusitis. Allergy Rhinol (Providence) 2012;3:e8-e12

13 McCaig LF, Hughes JM. Trends in antimicrobial drug prescribing among office-based physicians in the United States. JAMA 1995; 273:214-219

14 Williams JW Jr, Aguilar C, Makela M, et al. Antibiotics for acute maxillary sinusitis (Review). Cochrane Database Syst Rev 2000;2: CD000243

15 Lemiengre MB, van Driel ML, Merenstein D, Young J, De Sutter AI. Antibiotics for clinically diagnosed acute rhinosinusitis in adults. Cochrane Database Syst Rev 2012;10:CD006089

16 Chaturvedi J, Dutt SN, Kadambi P, et al. Factors that delay definitive management of chronic rhinosinusitis in India: a survey based study. Glob J Otolaryngol. 2016;2:555586

17 Witt C, Keil T, Selim D, et al. Outcome and costs of homoeopathic and conventional treatment strategies: a comparative cohort study in patients with chronic disorders. Complement Ther Med 2005;13:79-86

18 Esmail N. Complementary and Alternative Medicine: Use and Public Attitudes 1997, 2006, and 2016. Vancouver, BC: Frasser Institute; 2017

19 Barnes PM, Bloom B, Nahin RL. Complementary and alternative medicine use among adults and children: United States, 2007. Natl Health Stat Rep 2008;12:1-23

20 Krouse HJ, Krouse JH. Complementary therapeutic practices in patients with chronic sinusitis. Clin Excell Nurse Pract 1999; 3:346-352

21 Rotenberg BW, Bertens KA. Use of complementary and alternative medical therapies for chronic rhinosinusitis: a Canadian perspective. J Otolaryngol Head Neck Surg 2010;39:586-593

22 Suh JD, Wu AW, Taw MB, Nguyen C, Wang MB. Treatment of recalcitrant chronic rhinosinusitis with integrative East-West medicine: a pilot study. Arch Otolaryngol Head Neck Surg 2012;138:294-300

23 Wu AW, Gettelfinger JD, Ting JY, Mort C, Higgins TS. Alternative therapies for sinusitis and rhinitis: a systematic review utilizing a modified Delphi method. Int Forum Allergy Rhinol 2020;10:496-504
24 Jin AJ, Chin CJ. Complementary and alternative medicine in chronic rhinosinusitis: a systematic review and qualitative analysis. Am J Rhinol Allergy 2019;33:194-202

25 Friese KH, Zabalotnyi DI. Homeopathy in acute rhinosinusitis: a double-blind, placebo controlled study shows the efficiency and tolerability of a homeopathic combination remedy [article in German]. HNO 2007;55:271-277

26 Zabolotnyi DI, Kneis KC, Richardson A, et al. Efficacy of a complex homeopathic medication (Sinfrontal) in patients with acute maxillary sinusitis: a prospective, randomized, double-blind, placebocontrolled, multicenter clinical trial. Explore (NY) 2007;3:98-109

27 Weber U, Luedtke R, Friese KH, Fischer I, Moeller H. A nonrandomised pilot study to compare complementary and conventional treatments of acute sinusitis. Forsch Komplementarmed Klass Naturheilkd 2002;9:99-104

28 Kneis KC, Gandjour A. Economic evaluation of sinfrontal in the treatment of acute maxillary sinusitis in adults. Appl Health Econ Health Policy 2009;7:181-191

29 Heidbreder D, Gerster G, Gracza E. Sinusitis-klinische prüfung verschiedener homöopathischer medikamente in unterschiedlichen potenzen. Therapeutikon 1988;2:701-706

30 Heidl R. Statistische auswertung einer anwendungsbeobachtung mit SANUVIS Tropfen. SANUM Post 2009;86:23-25

31 Schmidt S, Kaestle HJ, Steinhausen U. Homeopathic spagyric therapy of acute and uncomplicated rhinosinusitis: an observational trial on symptom severity in 2 general practitioner surgeries. Forsch Komplement Med 2016;23:303-305

32 Adler M. Efficacy and safety of a fixed-combination homeopathic therapy for sinusitis. Adv Ther 1999;16:103-111

33 Ammerschläger $\mathrm{H}$, Klein $\mathrm{P}$, Weiser $\mathrm{M}$, Oberbaum M. Treatment of inflammatory diseases of the upper respiratory tract-comparison of a homeopathic complex remedy with xylometazoline [article in German]. Forsch Komplementarmed Klass Naturheilkd 2005;12:24-31

34 Suter A, Bommer S. Acute and chronic sinusitis: treatment with a homeopathic sinus spray. Schweiz Z Ganzheitsmed 2003; $15: 233-238$

35 Weiser M, Clasen BPE. Randomisierte lazebokontrollierte Doppelblindstudie zur Untersuchung der klinischen Wirksamkeit der homöopathischen Euphorbium compositum-Nasentropfen $S$ bei chronischer Sinusitis. Forsch Komplementarmed 1994; 1:251-259

36 Wiesenauer M, Gaus W, Bohnacker U, Häussler S. Efficiency of homeopathic preparation combinations in sinusitis. Results of a randomized double blind study with general practitioners [article in German]. Arzneimittelforschung 1989;39:620-625

37 Ricciotti F, Bernardini C, D'Aco L, et al. Studio pilota sulle rinosinusiti croniche per valutare l'efficacia e la tollerabilità di un trattamento antibiotico standard (Amoxicillina e acido Clavulanico) associato a un complesso omeopatico (Dr. Reckeweg R1). Rivista Ital Otolaringol Audiol Foniatria 2005;25:109-117

38 Nayak C, Singh V, Singh VP, et al. Homeopathy in chronic sinusitis: a prospective multi-centric observational study. Homeopathy 2012;101:84-91

39 Sharma SR, Bhanu KM, Sahagal GC, et al. Clinical evaluation of homoeopathic medicines in sinusitis. Indian J Res Homeopathy 2008;2:26-37

40 Ramteke SS, Nayak C, Singh V, et al. An open clinical observational study on the usefulness of pre-defined homoeopathic medicines in the management of chronic sinusitis. Indian J Res Homoeopathy 2009;3:34-40

41 Witt CM, Lüdtke R, Willich SN. Homeopathic treatment of patients with chronic sinusitis: a prospective observational study with 8 years follow-up. BMC Ear Nose Throat Disord 2009;9:7

42 Manchanda RK. A randomised, double blind, placebo-controlled, multi-centric parallel arm trial to assess the effects of homoeopathic medicines on chronic rhinosinusitis. Indian J Res Homoeopathy $2014 ; 8: 123-128$ 
43 Pynnonen MA, Kim HM, Terrell JE. Validation of the sino-nasal outcome test 20 (SNOT-20) domains in nonsurgical patients. Am J Rhinol Allergy 2009;23:40-45

44 EuroQol Research Foundation. EQ-5D-5L User Guide, 2019. Available at: https://euroqol.org/publications/user-guides. Accessed January 29, 2018

45 McCaffery M, Beebe A. Pain: Clinical manual for nursing practice. Mosby St. Louis, MO; 1989. J Pain Symptom Manage 1990;5:338-339

46 Jund R, Mondigler M, Stammer H, Stierna P, Bachert C. Herbal drug BNO 1016 is safe and effective in the treatment of acute viral rhinosinusitis. Acta Otolaryngol 2015;135:42-50

47 Schulz KF, Altman DG, Moher D; CONSORT Group. CONSORT 2010 statement: updated guidelines for reporting parallel group randomised trials. BMJ 2010;340:c332

48 Dean ME, Coulter MK, Fisher P, Jobst K, Walach H. Reporting data on homeopathic treatments (RedHot): a supplement to CONSORT. Homeopathy 2007;96:42-45
49 Banerjee A, Srivastava BP, Koley M, Saha S. An open-label observational trial to evaluate the possible effects of individualized homeopathic medicines in symptomatic nasal polyp. Bengal J Otolaryngol Head Neck Surg 2019;27:51-59

50 Vithoulkas G. Serious mistakes in meta-analysis of homeopathic research. J Med Life 2017;10:47-49

51 Coulter HL. Homoeopathy and modern medical science. The Hahn Glean 1982;49:147-166

52 Vithoulkas G. Talks on Classical Homoeopathy. New Delhi: B. Jain Publishers Pvt. Ltd.; 2013:234-240

53 Peters D, Taylor Reilly D, Feruguson A, et al. How I would treat chronic sinusitis. Complement Ther Med 1993;1:81-87

54 Nangia S, Giridher V, Chawla P. Evaluation of the role of nasal endoscopy and computed tomography individually in the diagnosis of chronic rhinosinusitis. Indian J Otolaryngol Head Neck Surg 2019;71:1711-1717 